\title{
Chiral condensate with topological degeneracy in graphene and its manifestation in edge states
}

\author{
Yuji Hamamoto, ${ }^{1}$ Hideo Aoki, ${ }^{2}$ and Yasuhiro Hatsugai ${ }^{1,3,}$ \\ ${ }^{1}$ Institute of Physics, University of Tsukuba, Tsukuba 305-8571, Japan \\ ${ }^{2}$ Department of Physics, University of Tokyo, Hongo, Tokyo 113-0033, Japan \\ ${ }^{3}$ Tsukuba Research Center for Interdisciplinary Material Science, University of Tsukuba, Tsukuba 305-8571, Japan
}

(Received 29 May 2012; revised manuscript received 4 October 2012; published 21 November 2012)

\begin{abstract}
The role of chiral symmetry in many-body states of graphene in strong magnetic fields is theoretically studied with the honeycomb lattice model. For a spin-split Landau level where the leading electron-electron interaction is the nearest-neighbor repulsion, a chiral condensate is shown to be, within the subspace of the $n=0$ Landau level, an exact many-body ground state having a finite gap, for which a calculation of the Chern number reveals that the ground state is a Hall insulator with a topological degeneracy of two. The topological nature of the ground state is shown to manifest itself as a Kekuléan bond order along armchair edges, while the pattern melts in the bulk due to quantum fluctuations. These can be regarded as a realization of the bulk-edge correspondence that is peculiar to a chiral-symmetric system. We have also obtained the ground state when point defects are introduced in the honeycomb lattice to reveal how the presence or absence of the chiral symmetry affects the defect states.
\end{abstract}

DOI: 10.1103/PhysRevB.86.205424

PACS number(s): 73.22.Pr, 71.10.Fd, 73.43.-f

\section{INTRODUCTION}

While the physics of graphene started from the one-body electronic structure as a Dirac fermion, a possible relevance of electron correlation in graphene has been intensively studied after a gap opening in the $n=0$ Landau level (LL) was experimentally observed in strong magnetic fields. ${ }^{1-4}$ Since it is difficult to explain the gap within a simple one-body problem, considerable theoretical efforts have ensued to clarify many-body effects in graphene quantum Hall $(\mathrm{QH})$ systems. ${ }^{5-18}$ However, little attention has been paid on how many-body effects should reflect the chiral symmetry in the graphene $\mathrm{QH}$ regime, which is, after all, a fundamental symmetry inherent in graphene's honeycomb lattice. On the one-body level, the effects of chiral symmetry in graphene is well understood: To start with, the symmetry guarantees the emergence of doubled Dirac cones in the Brillouin zone, which can be interpreted as a two-dimensional analog of the NielsenNinomiya theorem that is well known in four-dimensional lattice gauge theory. We can even examine the wave functions in terms of the Aharonov-Casher argument, which states that chiral symmetry topologically protects the degeneracy of the $n=0 \mathrm{LL}$ against random gauge fields. ${ }^{19} \mathrm{~A}$ similar situation occurs for ripples in a graphene sheet, which can be modeled by random hopping amplitudes. Kawarabayashi et al. have shown that the $n=0$ LL exhibits an anomalously sharp (deltafunction-like) density of states (DOS) as soon as the spatial wavelength of the ripple exceeds a few lattice constants. ${ }^{20}$

In the presence of electron-electron interactions, on the other hand, the role of chiral symmetry has been investigated primarily in zero magnetic fields in the context of spontaneous symmetry breaking. ${ }^{8,21-23}$ While these studies mainly employ a Dirac field model in a continuum space to discuss many-body gap formation, such an effective treatment may well overlook the essence of graphene's chiral symmetry, which is intimately related to the underlying honeycomb lattice.

With this background, we shed light in the present paper on how the chiral symmetry influences the many-body problem in the graphene QH effect, by fully taking account of the lattice structure. We first examine the many-body problem with exact diagonalization in a subspace projected into the $n=0 \mathrm{LL}$. Working on the subspace enables us to classify many-body states according to a notion of the total chirality of the filled zero modes. In terms of this, for a "bipartite" electron-electron interaction such as the nearest-neighbor repulsion, which is the dominant interaction for a spin-split LL, we show that the many-body ground state is exactly identified to be a chiral condensate with a topological degeneracy of two. We confirm numerically that there exists a finite energy gap to the first excited state, which makes the Chern number of the ground state well defined. The total Chern number contributed by the filled zero modes along with the negative energy states ("Dirac sea") turns out to be zero, which implies the system is a Hall insulator with vanishing Hall conductance.

Despite the cancellation of the Chern number in the bulk, however, we move on to show that the topological nature of the chiral condensate is in fact made manifest as an emergence of a Kekuléan bond order in the edge state along the armchair edges of the honeycomb lattice (in sharp contrast to zigzag edge states in the one-body problem). On the mean-field (MF) level the Kekulé pattern is shown to appear in the bulk with a Kekuléan degeneracy of three, so that the present result amounts that the mean-field order, while dissolved in the topologically degenerate chiral condensate in the bulk, resurfaces along an armchair edge. This can be interpreted as an example of bulk-edge correspondence, ${ }^{24}$ which states that a topologically nontrivial bulk state should always accompany a characteristic edge state. In the case of graphene, the bulk-edge correspondence becomes peculiar in that the chiral condensate manifests itself as a freezing of a Kekuléan bond order along a specific (armchair) edges. We also obtain the ground state when point defects are introduced in the honeycomb lattice to discuss how the chiral symmetry affects the defect states.

\section{SPINLESS MODEL}

To model interacting electrons on a honeycomb lattice, here we consider spin-polarized electrons, ${ }^{25}$ so that the leading Coulomb interaction reduces to the nearest-neighbor 
repulsion. The Hamiltonian then reads $\mathcal{H}=\mathcal{H}_{\text {kin }}+\mathcal{H}_{\text {int }}$, where the kinetic term

$$
\mathcal{H}_{\text {kin }}=-t \sum_{\langle i j\rangle}\left(e^{i \theta_{i j}} c_{i}^{\dagger} c_{j}+\text { H.c. }\right) \equiv c^{\dagger} H_{\text {kin }} c
$$

describes hopping between adjacent sites $\langle i j\rangle$ with strength $t>0$. The magnetic field is included as the Peierls phase $\theta_{i j}$ such that magnetic flux per elementary hexagon equals $\sum_{\square} \theta_{i j}=2 \pi \phi$ in units of a magnetic flux quantum $h / e$. For a honeycomb lattice with $N_{\bullet} \circ$ s) sites in sublattice $\bullet(\circ)$, $c^{\dagger}=\left(c_{0}^{\dagger}, c_{\circ}^{\dagger}\right)$ with $c_{\bullet(\circ)}^{\dagger}$ a row of creation operators for sublattice -(०) and $H_{\text {kin }}$ is a square matrix of dimension $N_{\bullet}+N_{\circ}$. If we introduce $\Gamma=\operatorname{diag}\left(I_{\bullet},-I_{\circ}\right)$ with an identity matrix $I_{\bullet(\circ)}$ of dimension $N_{\bullet(\circ)}$, the kinetic term satisfies an anticommutation relation $\left\{H_{\mathrm{kin}}, \Gamma\right\}=0$, which defines the chiral symmetry. The symmetry implies that, if $\psi_{k}$ is the $k$ th eigenvector with energy $\varepsilon_{k}$, a chiral partner $\Gamma \psi_{k}$ exists with an energy $-\varepsilon_{k}$. This makes the $n=0$ LL special in that we can take $\psi_{k}$ as an eigenstate of $\Gamma$ as $\Gamma \psi_{k \pm}= \pm \psi_{k \pm}$. The interaction between spin-polarized electrons is expressed in a particle-hole symmetric form as

$$
\begin{aligned}
\mathcal{H}_{\text {int }} & =\sum_{i \neq j} V_{i j}\left(n_{i}-\frac{1}{2}\right)\left(n_{j}-\frac{1}{2}\right) \\
& =\frac{1}{2} \sum_{i \neq j} V_{i j}\left(c_{i}^{\dagger} c_{j}^{\dagger} c_{j} c_{i}+c_{i} c_{j} c_{j}^{\dagger} c_{i}^{\dagger}\right)+\text { const },
\end{aligned}
$$

where $V_{i j}$ is the strength of the electron-electron interaction, and $n_{i} \equiv c_{i}^{\dagger} c_{i}$ is the number operator at site $i$.

\section{CHIRAL CONDENSATE}

We start with an investigation of the many-body problem at half filling. Since it is difficult to treat all of the many-body states exactly, we shrink the Hilbert space by assuming that the states are reconstructed within the $n=0 \mathrm{LL}$ while the negative-energy states are kept fully filled. Such a treatment is valid as long as $\left|V_{i j}\right|$ is perturbatively small compared with the Landau gaps around the $n=0 \mathrm{LL}{ }^{26}$ In the $n=0$ LL, we take a zero mode multiplet $\psi=\left(\psi_{+}, \psi_{-}\right)$where we have decomposed it into eigenstates of the chiral operator, $\psi_{ \pm}=\left(\psi_{1 \pm}, \ldots, \psi_{M_{ \pm} \pm}\right)$with degeneracy $M_{ \pm}$. Note that the zero modes are localized on each of the sublattices as $\psi_{+}=\frac{1}{\sqrt{2}}\left(\begin{array}{c}\psi_{\bullet} \\ 0\end{array}\right)$ and $\psi_{+}=\frac{1}{\sqrt{2}}\left(\begin{array}{c}0 \\ \psi_{0}\end{array}\right)$. If we introduce the negativeenergy multiplet $\varphi=\left(\varphi_{1}, \varphi_{2}, \ldots\right)$ such that $H_{\text {kin }} \varphi_{k}=\varepsilon_{k} \varphi_{k}$ with $\varepsilon_{k}<0,\left(\psi_{+}, \psi_{-}, \varphi, \Gamma \varphi\right)$ forms a complete set, so that the fermion operator is expanded as $c=\psi d+\varphi d_{<}+\Gamma \varphi d_{>}$, where $d=\left(\begin{array}{c}d_{+} \\ d_{-}\end{array}\right)$and $d_{\lessgtr}$ are columns of fermion operators for the zero modes and the $\varepsilon \lessgtr 0$ states, respectively. In the projected subspace, the Hamiltonian is reduced, up to a constant, to $^{27}$

$$
\tilde{\mathcal{H}}=\frac{1}{2} \sum_{i \neq j} V_{i j}\left(\tilde{c}_{i}^{\dagger} \tilde{c}_{j}^{\dagger} \tilde{c}_{j} \tilde{c}_{i}+\tilde{c}_{i} \tilde{c}_{j} \tilde{c}_{j}^{\dagger} \tilde{c}_{i}^{\dagger}\right)
$$

where the projected fermion operator, $\tilde{c}_{i} \equiv(\psi d)_{i}$, no longer satisfies the canonical anticommutation relations (see Appendix A). Still, the generator of the total chirality of the filled zero modes can be defined as

$$
\mathcal{G}=\tilde{c}^{\dagger} \Gamma \tilde{c}=d_{+}^{\dagger} d_{+}-d_{-}^{\dagger} d_{-} .
$$

Due to the invariance of $\tilde{\mathcal{H}}$ for the chiral transformation $\tilde{\mathcal{H}} \mapsto e^{i \theta \mathcal{G}} \tilde{\mathcal{H}} e^{-i \theta \mathcal{G}}=\tilde{\mathcal{H}}$, the total chirality is conserved,

$$
[\tilde{\mathcal{H}}, \mathcal{G}]=0 .
$$

Then the many-body states are classified according to the total chirality $\chi_{\text {tot }}$. For repulsive interactions $V_{i j}>0, \tilde{\mathcal{H}}$ is semipositive definite. Furthermore, when the interactions are bipartite (i.e., only act between different sublattices,

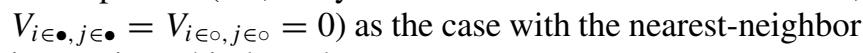
interaction, chiral condensates

$$
\left|G_{ \pm}\right\rangle=d_{1 \pm}^{\dagger} \cdots d_{M_{ \pm} \pm}^{\dagger}\left|D_{<}\right\rangle
$$

with the Dirac sea $\left|D_{<}\right\rangle \equiv \prod_{k}\left(c^{\dagger} \varphi\right)_{k}|0\rangle$ constitute a ground-state doublet $\Psi=\left(\left|G_{+}\right\rangle,\left|G_{-}\right\rangle\right)$, since $\tilde{c}_{j} \tilde{c}_{i}\left|G_{ \pm}\right\rangle=$ $\tilde{c}_{j}^{\dagger} \tilde{c}_{i}^{\dagger}\left|G_{ \pm}\right\rangle=0$ for, e.g., $i \in \bullet$ and $j \in 0^{26,28} \mathrm{We}$ call this a topological degeneracy of two. Unlike a simple charge density wave (CDW), one may mix $\left|G_{+}\right\rangle$and $\left|G_{-}\right\rangle$through a unitary transformation $\Psi \mapsto \Psi^{\omega} \omega$ with $\omega \in U(2)$ even in a finite system. One can numerically confirm that the chiral condensate remains the ground state unless the nonbipartite potential is large, ${ }^{12}$ even though the ground-state energy is nonzero. In the rest of the paper, we focus on the leading interaction, i.e., the nearest-neighbor repulsion for simplicity.

\section{MANY-BODY GAP}

We next calculate excitation energies numerically with the exact diagonalization method (see Appendix B). In the projected subspace, the strength of the nearest-neighbor repulsion $V>0$ is the only energy scale, which acts as the unit of energy. Full energy spectra of $\tilde{\mathcal{H}}$ for finite systems suggest that the first excited state appears in the sector of $\chi_{\text {tot }}= \pm\left(M_{ \pm}-2\right),{ }^{12}$ which is created from a chiral condensate $\left|G_{ \pm}\right\rangle$by single chirality flippings analogous to the projected single-mode approximation. ${ }^{29-31}$ Noticing this, we further shrink the Hilbert space by focusing on the sector of $\chi_{\text {tot }}= \pm\left(M_{ \pm}-2\right)$. This enables us to obtain the energy of the first excited state, or the energy gap $\Delta$, with a calculation cost of the order of $O\left(M_{ \pm}^{2}\right)$. We consider a system on a torus composed of $2 L^{2}$ lattice sites with a linear dimension $L$. For investigating a weak-field regime, we adopt the string gauge, ${ }^{32}$ where a magnetic flux is given by $\phi=m / L^{2}$ with an integer $m(>0)$ and $M_{ \pm}=m$ zero modes are obtained for each chirality.

In Fig. 1, we plot $\Delta$ for 30 electrons as a function of $\phi$ with $L$ changed consecutively. We immediately notice that the result exhibits a marked periodicity of three, where the values for $L \equiv 3(\bmod 3)$ form a clear lower envelope with a scaling $\Delta \propto \phi^{2}$, while those for $L \neq 3 l$ deviate from this. The latter behavior is considered to be a finite-size effect, since the deviation diminishes with the sample size. To confirm the scaling, the inset plots $\Delta / \phi^{2}$ at $L=3 l$ against $1 / L$ for various values of $\phi$, which indicates the scaling law $\Delta \propto \phi^{2}$ is very accurately obeyed.

\section{HALL CONDUCTANCE}

Let us now consider the Hall conductance of the chiral condensate. By the Niu-Thouless-Wu formula, ${ }^{33}$ the Hall 


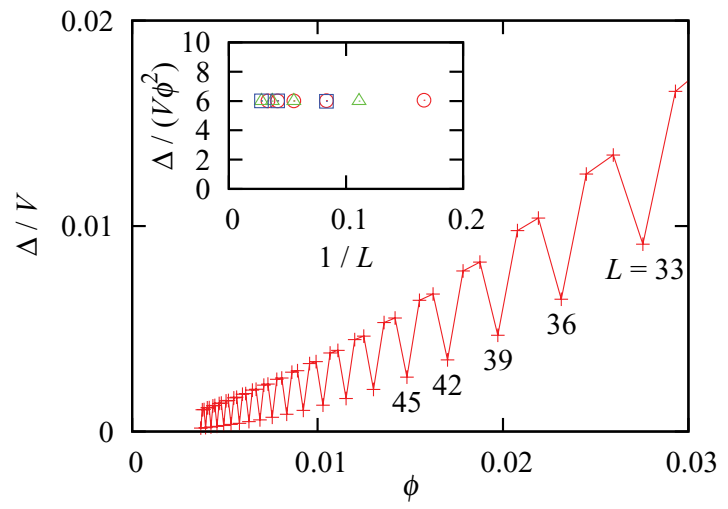

FIG. 1. (Color online) Magnetic-field dependence of the manybody gap $\Delta$ in the $n=0 \mathrm{LL}$. The result is displayed for 30 electrons with the consecutive values of the linear dimension of the system $L$ connected by a line with the results for $L \equiv 3(\bmod 3)$ forming a lower envelope $\left(\Delta \propto \phi^{2}\right)$. The inset shows the gap at $L=3 l$ against $1 / L$ for the magnetic flux $\phi=1 / 12$ (circle), $\phi=1 / 27$ (triangle), and $\phi=1 / 48$ (square).

conductance is written with the Chern number ${ }^{34}$ as

$$
\begin{gathered}
\sigma_{x y}=\frac{e^{2}}{h} \frac{1}{N_{D}} C \\
C=\frac{1}{2 \pi i} \int \operatorname{Tr}_{N_{D}} d A \quad\left(A=\Psi^{\dagger} d \Psi\right),
\end{gathered}
$$

where $N_{D}$ is the degeneracy and $A$ is the non-Abelian Berry connection that describes multiplets. ${ }^{35}$ In terms of the basis that diagonalizes $\mathcal{G}$, we have $C=C_{+}+C_{-}$with

$$
C_{ \pm}=\frac{1}{2 \pi i} \int\left\langle d G_{ \pm} \mid d G_{ \pm}\right\rangle .
$$

Each Chern number is further decomposed as $C_{ \pm}=C_{\psi_{ \pm}}+$ $C_{D_{<}}$with

$$
\begin{gathered}
C_{\psi_{ \pm}}=\frac{1}{2 \pi i} \int \operatorname{Tr}_{M_{ \pm}} d \psi_{ \pm}^{\dagger} d \psi_{ \pm}, \\
C_{D_{<}}=\frac{1}{2 \pi i} \int \operatorname{Tr} d \varphi^{\dagger} d \varphi .
\end{gathered}
$$

By the charge conjugation, we have $C_{\psi_{+}}+C_{D_{<}}=-\left(C_{\psi_{-}}+\right.$ $C_{D_{>}}$), where we can show that

$$
C_{D_{>}}=\frac{1}{2 \pi i} \int \operatorname{Tr}(\Gamma d \varphi)^{\dagger} \Gamma d \varphi=C_{D_{<}} .
$$

Thus the total Chern number of the ground-state doublet vanishes as

$$
C=C_{\psi_{+}}+C_{\psi_{-}}+2 C_{D_{<}}=0,
$$

which may be called a topological cancellation. This implies that the chiral condensate is a Hall insulator with a nontrivial topological degeneracy $N_{D}=2$.

\section{BOND ORDER}

As have been confirmed in various systems, while topological phases are featureless in a bulk, they show characteristic boundary states. ${ }^{24}$ So a natural question we can pose here is as follows: Do the edge states in the present system exhibit special features despite the bulk Chern number being zero? Before presenting the result, however, let us first have a look (a)
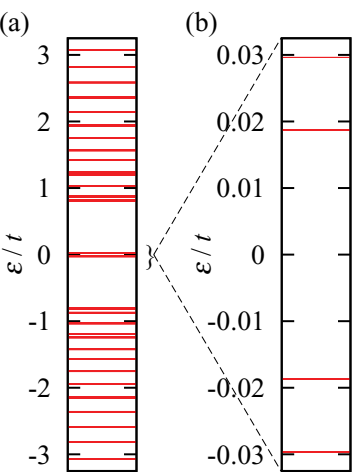

(c)

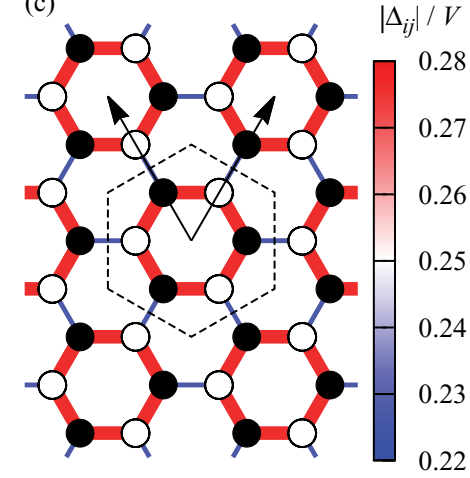

FIG. 2. (Color online) Mean-field results for the energy spectrum (a), its blowup around the $n=0 \mathrm{LL}$ (b), and the bond strength $\left|\Delta_{i j}\right|$ plotted in a real space (c). The parameters are $L=15, \phi=1 / 15$, and $V / t=0.25$. A dashed hexagon in (c) is an enlarged unit cell with arrows primitive vectors.

at the mean-field state in the present system in the bulk, which will turn out to be instructive. One virtue of a mean-field picture $^{36}$ is that we can introduce a bond order,

$$
\Delta_{i j} \equiv V\left\langle c_{i}^{\dagger} c_{j}\right\rangle
$$

for adjacent sites $\langle i j\rangle .^{37-41}$ The dominant part of the MF Hamiltonian is given by

$$
\mathcal{H}_{\mathrm{MF}}=-\sum_{\langle i j\rangle}\left[\left(t e^{i \theta_{i j}}+\Delta_{i j}^{*}\right) c_{i}^{\dagger} c_{j}+\text { H.c. }\right],
$$

where $\Delta_{i j}$ is determined self-consistently by diagonalizing $\mathcal{H}_{\text {MF }}$. A spontaneous symmetry breaking is induced by the many-body effect for weak magnetic fields, where the density of states has a sharp peak at the Fermi energy. In Fig. 2, we show a typical MF result for the ordered phase. The energy spectrum is plotted in Fig. 2(a), where the qualitative structure of the LLs is preserved. This comes from the fact that the convergent order parameters turn out to retain the initial Peierls phase as $\Delta_{i j}=\left|\Delta_{i j}\right| e^{-i \theta_{i j}}$. The influence of the electron-electron interaction appears most prominently in the $n=0 \mathrm{LL}$, where a finite gap of the order of $\phi$ opens as shown in the blowup in Fig. 2(b). To see how the symmetry is broken in the mean field, we show in Fig. 2(c) a real-space image of the bond order $\left|\Delta_{i j}\right|$, which is seen to exhibit a Kekulé pattern. ${ }^{36,42}$ This makes the unit cell enlarged, which causes $K$ and $K^{\prime}$ points to be coupled, and this in turn opens a finite gap. In this sense we can regard this as a Peierls transition in the honeycomb lattice.

On the other hand, the chiral condensate with its topological degeneracy of two does not exhibit bond order in the bulk as we have seen in Fig. 2. Due to the quantum fluctuation, the bond order of the mean field is destroyed and the quantum liquid ground state is realized.

\section{EDGE AND DEFECT STATES}

We are now in a position to ask the following question: What kind of edge state does the chiral condensate accommodate? Based on the bulk-edge correspondence, we may expect a nontrivial behavior of the many-body states near the edges. A prime example is the fractional QH states in a two-dimensional 

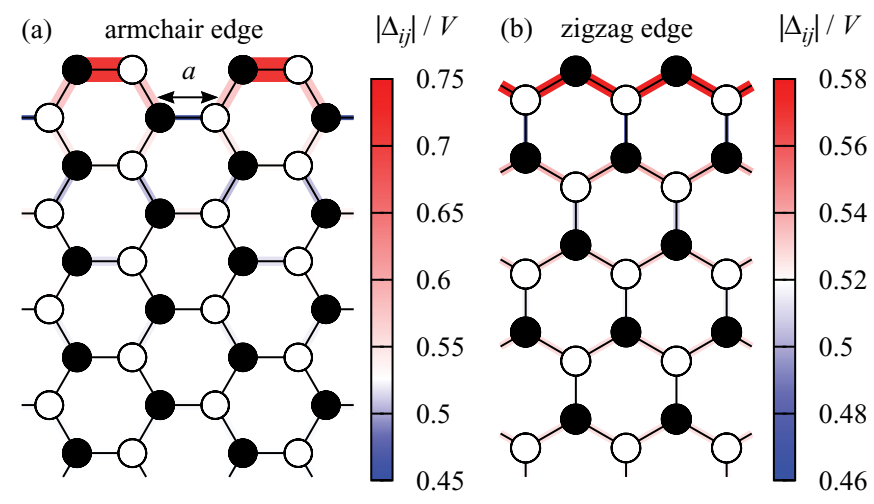

FIG. 3. (Color online) Bond strength near an armchair (left) or zigzag (right) edge of the doubly degenerate chiral condensate. The magnetic flux is $\phi=1 / 192$ for which the magnetic length is $l_{B} \simeq$ 8.9a. The bond order decays in a bulk away from the edges.

electron gas (2DEG), where a CDW-like behavior emerges along the edges of a ribbon, ${ }^{43}$ while in the bulk it melts into the Laughlin liquid with no long-range order, which has a $q$-fold degeneracy of the fractional $\mathrm{QH}$ states at filling $v=1 / q$. Note that a honeycomb lattice with edges has $\mathrm{QH}$ edge states whose mode lies in a LL gap. To perform the projection into the $n=0$ LL we set an energy cutoff, the choice of which is shown to have little influence on the edge states shown below.

In Fig. 3 we show $\left|\Delta_{i j}\right|$ for the chiral condensate plotted in a real space near the armchair and zigzag edges. In Fig. 3(a), we can see that a Kekule-type bond order that is reminiscent of the mean-field result in Fig. 2 emerges along the armchair edge. This is the key result in the present work. The enhancement in bonds rapidly decays away from the edge in a few lattice constants, and $\left|\Delta_{i j}\right|$ slightly oscillates with a length scale of the order of the magnetic length $l_{B} \sim a / \sqrt{\phi}$, with $a$ being the interatomic spacing. This may naively seem to be analogous to the fractional $\mathrm{QH}$ edge states in a $2 \mathrm{DEG}$, but here the honeycomb lattice structure is essential in the ground state. Indeed, the ring pattern is locked along the armchair edge in a Kekule pattern, while this is not the case with zigzag edges [see Fig. 3(b)]. In the latter case, the ring pattern is blurred by the translational symmetry along a zigzag edge, and a very weak stripe pattern parallel to the edge appears. These patterns related with the threefold degeneracy of the Kekulé pattern are washed out in the bulk chiral condensate. All these are a specific property of a honeycomb lattice model.

We can further endorse that the lattice structure is at the core by looking at the states around lattice defects. When a single atom is removed from the bulk honeycomb lattice, one-body localized zero modes appear that are protected by the chiral symmetry. ${ }^{44,45}$ In the presence of the electron-electron interactions, however, local chiral symmetry breaking occurs spontaneously to lower the energy by inducing effective hopping in the same sublattice. ${ }^{45,46}$ Then what if two point defects come close to each other? Such a divacancy consists of two adjacent missing atoms, and is recently observed experimentally in ion-irradiated carbon samples. ${ }^{47}$ We expect that the chiral symmetry may be partially recovered with a reconfiguring of the two symmetry-breaking bonds. We plot in Fig. 4 the bond order for the chiral condensate near the

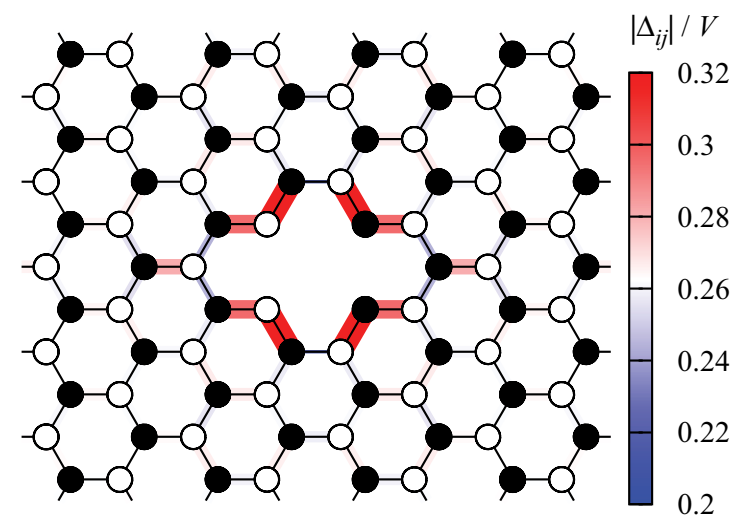

FIG. 4. (Color online) Bond strength of the chiral condensate near a divacancy composed of two adjacent sites that are missing. The magnetic flux is $\phi=1 / 1200$ for which the magnetic length is $l_{B} \simeq 22.3 a$. The bond order reflects twofold axial symmetry of the divacancy.

divacancy. We do confirm that enhancement of the bond order near the divacancy, which can be considered due to the revival of the chiral symmetry.

We have thus shown that the bond order emerges along edges and around vacancies, despite the topological cancellation $C=0$ that might first seem to wipe out any signature of the chiral condensate. Indeed, the charge density $\left\langle n_{i}\right\rangle$ itself is uniform for the chiral condensate even along the edges, which is due to the invariance of the chiral condensate for the charge conjugation. Thus it is the bond order $\left|\Delta_{i j}\right|$ that we have to look at as a probe for the chiral condensate. Thus the bond order provides a probe for the many-body effect in half-filled graphene in a magnetic field. The bond order near the edges should be observable experimentally with some imaging techniques such as Green's function scanning tunneling microscopes. ${ }^{48,49}$ Since the amplitude of $\left|\Delta_{i j}\right| / V=\left|\left\langle c_{i}^{\dagger} c_{j}\right\rangle\right|$ is of the order of the magnetic flux $\phi$, the magnetic field should have significant magnitudes.

\section{SUMMARY}

The many-body ground state at half filling in the honeycomb lattice is identified as a doubly degenerate chiral condensate for a spin-split Landau level. The many-body effect opens a finite energy gap, which makes the chiral condensate a generic topological insulator. However, the system has a peculiar manifestation of the bulk-edge correspondence in topological systems as an emergence of a bond order with a Kekulé pattern along armchair edges in an exact ground state, while the pattern is dissolved in the bulk.

\section{ACKNOWLEDGMENTS}

The computation in this work has been done with the facilities of the Supercomputer Center, Institute for Solid State Physics, University of Tokyo. This work was supported in part by Grants-in-Aid for Scientific Research No. 23340112 and No. 23654128 from the JSPS. 


\section{APPENDIX A: ANTICOMMUTATION RELATIONS IN THE $n=0$ LANDAU LEVEL}

We show how the anticommutation relations are modified by the projection into the $n=0 \mathrm{LL}$. From the canonical anticommutation relations between $c_{i}$ 's, it is clear that $d_{\alpha \chi} \equiv$ $\psi_{\alpha \chi}^{\dagger} c$, where $\chi= \pm$ and $\alpha$ labels the zero modes with the chirality, is a canonical fermion as

$$
\begin{gathered}
\left\{d_{\alpha \chi}, d_{\beta \epsilon}^{\dagger}\right\}=\sum_{i j}\left(\psi_{\alpha \chi}\right)_{i}^{*}\left(\psi_{\beta \epsilon}\right)_{j}\left\{c_{i}, c_{j}^{\dagger}\right\}=\delta_{\alpha \beta} \delta_{\chi \epsilon}, \\
\left\{d_{\alpha \chi}, d_{\beta \epsilon}\right\}=\sum_{i j}\left(\psi_{\alpha \chi}\right)_{i}^{*}\left(\psi_{\beta \epsilon}\right)_{j}^{*}\left\{c_{i}, c_{j}\right\}=0, \\
\left\{d_{\alpha \chi}^{\dagger}, d_{\beta \epsilon}^{\dagger}\right\}=0 .
\end{gathered}
$$

On the other hand, $\tilde{c} \equiv \psi d$ no longer obeys the fermionic anticommutation relations, but has

$$
\begin{gathered}
\left\{\tilde{c}_{i}, \tilde{c}_{j}^{\dagger}\right\}=\sum_{\alpha \beta \chi \epsilon}\left(\psi_{\alpha \chi}\right)_{i}\left(\psi_{\beta \epsilon}\right)_{j}^{*}\left\{d_{\alpha \chi}, d_{\beta \epsilon}^{\dagger}\right\}=\left(\psi \psi^{\dagger}\right)_{i j} \equiv p_{i j}, \\
\left\{\tilde{c}_{i}, \tilde{c}_{j}\right\}=\sum_{\alpha \beta \chi \epsilon}\left(\psi_{\alpha \chi}\right)_{i}\left(\psi_{\beta \epsilon}\right)_{j}\left\{d_{\alpha \chi}, d_{\beta \epsilon}\right\}=0 \\
\left\{\tilde{c}_{i}^{\dagger}, \tilde{c}_{j}^{\dagger}\right\}=0
\end{gathered}
$$

where the square matrix $p \equiv \psi \psi^{\dagger}$ is a projection into the $n=0 \mathrm{LL}$ as $\tilde{c}=p c$.

\section{APPENDIX B: PSEUDOPOTENTIAL}

If we restrict ourselves to the bipartite interactions, the projected Hamiltonian (4) is rewritten as

$$
\tilde{\mathcal{H}}=\frac{1}{2} \sum_{\alpha \beta \gamma \delta}\left(V_{\alpha \beta \gamma \delta} d_{\alpha+}^{\dagger} d_{\beta-}^{\dagger} d_{\gamma-} d_{\delta+}+V_{\alpha \beta \gamma \delta}^{*} d_{\alpha+} d_{\beta-} d_{\gamma_{-}-}^{\dagger} d_{\delta+}^{\dagger}\right),
$$

with the pseudopotential

$$
V_{\alpha \beta \gamma \delta}=\sum_{i \in \bullet, j \in \circ} V_{i j}\left(\psi_{\alpha+}\right)_{i}^{*}\left(\psi_{\beta-}\right)_{j}^{*}\left(\psi_{\gamma-}\right)_{j}\left(\psi_{\delta+}\right)_{i}=V_{\delta \gamma \beta \alpha}^{*} .
$$

Numerical calculations are performed using a basis

$$
\begin{aligned}
|\boldsymbol{p}, \boldsymbol{m}\rangle & =d_{p_{1}+}^{\dagger} \cdots d_{p_{N_{+}}+}^{\dagger} d_{m_{1}-}^{\dagger} \cdots d_{m_{N_{-}}}^{\dagger}|0\rangle, \\
p_{1} & <p_{2}<\cdots<p_{N_{+}}, \\
m_{1} & <m_{2}<\cdots<m_{N_{-}},
\end{aligned}
$$

where $\boldsymbol{p}=\left(p_{1}, \ldots, p_{N_{+}}\right)$and $\boldsymbol{m}=\left(m_{1}, \ldots, m_{N_{-}}\right)$. *hatsugai.yasuhiro.ge@u.tsukuba.ac.jp

${ }^{1}$ Y. Zhang, Z. Jiang, J. P. Small, M. S. Purewal, Y.-W. Tan, M. Fazlollahi, J. D. Chudow, J. A. Jaszczak, H. L. Stormer, and P. Kim, Phys. Rev. Lett. 96, 136806 (2006).

${ }^{2}$ Z. Jiang, Y. Zhang, H. L. Stormer, and P. Kim, Phys. Rev. Lett. 99, 106802 (2007).

${ }^{3}$ Y. Zhao, P. Cadden-Zimansky, F. Ghahari, and P. Kim, Phys. Rev. Lett. 108, 106804 (2012).

${ }^{4}$ A. F. Young, C. R. Dean, L. Wang, H. Ren, P. Cadden-Zimansky, K. Watanabe, T. Taniguchi, J. Hone, K. L. Shepard, and P. Kim, Nat. Phys. 8, 550 (2012).

${ }^{5}$ K. Nomura and A. H. MacDonald, Phys. Rev. Lett. 96, 256602 (2006).

${ }^{6}$ J. Alicea and M. P. A. Fisher, Phys. Rev. B 74, 075422 (2006).

${ }^{7}$ M. O. Goerbig, R. Moessner, and B. Douçot, Phys. Rev. B 74, 161407 (2006).

${ }^{8}$ V. P. Gusynin, V. A. Miransky, S. G. Sharapov, and I. A. Shovkovy, Phys. Rev. B 74, 195429 (2006).

${ }^{9}$ J.-N. Fuchs and P. Lederer, Phys. Rev. Lett. 98, 016803 (2007).

${ }^{10}$ I. F. Herbut, Phys. Rev. B 75, 165411 (2007).

${ }^{11}$ J. Alicea and M. P. A. Fisher, Solid State Commun. 143, 504 (2007).

${ }^{12}$ L. Sheng, D. N. Sheng, F. D. M. Haldane, and L. Balents, Phys. Rev. Lett. 99, 196802 (2007).

${ }^{13}$ J. Jung and A. H. MacDonald, Phys. Rev. B 80, 235417 (2009).

${ }^{14}$ K. Nomura, S. Ryu, and D.-H. Lee, Phys. Rev. Lett. 103, 216801 (2009).

${ }^{15}$ C.-Y. Hou, C. Chamon, and C. Mudry, Phys. Rev. B 81, 075427 (2010).

${ }^{16}$ Z. Yang and J. H. Han, Phys. Rev. B 81, 115405 (2010).

${ }^{17}$ I. F. Herbut, Phys. Rev. B 81, 205429 (2010).

${ }^{18}$ M. Kharitonov, Phys. Rev. B 85, 155439 (2012).
${ }^{19}$ Y. Aharonov and A. Casher, Phys. Rev. A 19, 2461 (1979).

${ }^{20}$ T. Kawarabayashi, Y. Hatsugai, and H. Aoki, Phys. Rev. Lett. 103, 156804 (2009).

${ }^{21}$ J. E. Drut and T. A. Lähde, Phys. Rev. Lett. 102, 026802 (2009).

${ }^{22}$ J. E. Drut and T. A. Lähde, Phys. Rev. B 79, 165425 (2009).

${ }^{23}$ Y. Araki and T. Hatsuda, Phys. Rev. B 82, 121403 (2010).

${ }^{24}$ Y. Hatsugai, Phys. Rev. Lett. 71, 3697 (1993).

${ }^{25}$ Along with the spin-polarized scenario at the charge neutrality point (Ref. 2), a spin-unpolarized one has also been proposed in recent experiments (Refs. 3 and 4). At any rate, the results obtained in the present paper have experimental relevance in the context of cold atoms on a optical lattice (Ref. 50).

${ }^{26}$ Y. Hatsugai, T. Morimoto, T. Kawarabayashi, Y. Hamamoto, and H. Aoki, arXiv: 1210.0714 .

${ }^{27}$ We have justified the projected Hamiltonian (4) by confirming that the energy spectrum scarcely changes from that obtained with unprojected Hamiltonian (3).

${ }^{28}$ Y. Hamamoto, Y. Hatsugai, and H. Aoki, arXiv:1108.1638 [Journal of Physics: Conference Series (to be published)].

${ }^{29}$ S. M. Girvin, A. H. MacDonald, and P. M. Platzman, Phys. Rev. Lett. 54, 581 (1985).

${ }^{30}$ S. M. Girvin, A. H. MacDonald, and P. M. Platzman, Phys. Rev. B 33, 2481 (1986).

${ }^{31}$ T. Nakajima and H. Aoki, Phys. Rev. Lett. 73, 3568 (1994).

${ }^{32}$ Y. Hatsugai, K. Ishibashi, and Y. Morita, Phys. Rev. Lett. 83, 2246 (1999).

${ }^{33}$ Q. Niu, D. J. Thouless, and Y.-S. Wu, Phys. Rev. B 31, 3372 (1985).

${ }^{34}$ D. J. Thouless, M. Kohmoto, M. P. Nightingale, and M. den Nijs, Phys. Rev. Lett. 49, 405 (1982).

${ }^{35}$ Y. Hatsugai, J. Phys. Soc. Jpn. 73, 2604 (2004).

${ }^{36}$ Y. Hatsugai, T. Fukui, and H. Aoki (unpublished). 
${ }^{37}$ I. Affleck and J. B. Marston, Phys. Rev. B 37, 3774 (1988).

${ }^{38}$ X. G. Wen, F. Wilczek, and A. Zee, Phys. Rev. B 39, 11413 (1989).

${ }^{39}$ J. Voit, Phys. Rev. B 45, 4027 (1992).

${ }^{40}$ M. Nakamura, Phys. Rev. B 61, 16377 (2000).

${ }^{41}$ B. Uchoa and A. H. Castro Neto, Phys. Rev. Lett. 98, 146801 (2007).

${ }^{42}$ Y. Hatsugai, T. Fukui, and H. Aoki, Physica E 40, 1530 (2008).

${ }^{43}$ E. H. Rezayi and F. D. M. Haldane, Phys. Rev. B 50, 17199 (1994).

${ }^{44}$ S. Ryu and Y. Hatsugai, Phys. Rev. Lett. 89, 077002 (2002).

${ }^{45}$ Y. Hatsugai, Solid State Commun. 149, 1061 (2009).
${ }^{46}$ S. Ryu and Y. Hatsugai, Physica E 22, 679 (2004).

${ }^{47}$ M. M. Ugeda, I. Brihuega, F. Hiebel, P. Mallet, J.-Y. Veuillen, J. M. Gómez-Rodríguez, and F. Ynduráin, Phys. Rev. B 85, 121402 (2012), to be precise, have detected five-membered rings adjacent to an eight-membered one, where odd-membered rings are expected to have significant effects on the chiral states.

${ }^{48}$ J. M. Byers and M. E. Flatté, Phys. Rev. Lett. 74, 306 (1995).

${ }^{49}$ Q. Niu, M. C. Chang, and C. K. Shih, Phys. Rev. B 51, 5502 (1995).

${ }^{50}$ J. Dalibard, F. Gerbier, G. Juzeliūnas, and P. Öhberg, Rev. Mod. Phys. 83, 1523 (2011). 